\title{
Research and Application of Dispatching Misoperation Prevention System for Integrated Dispatching and Controlling
}

\author{
Yuan-shuai Dong, Jian Cheng, Bin PENG \\ NARI Technology Development Co. Ltd., Nanjing 210061, China \\ dongyuanshuai@sgepri.sgcc.com.cn, chengjian@sgepri.sgcc.com.cn, \\ pengbin@sgepri.sgcc.com.cn
}

\begin{abstract}
Keywords: integrated dispatching and controlling; panoramic data; online misoperation prevention; hierarchical analysis.

Abstract. The possibility of dispatching misoperation under integrated dispatching and controlling and the defects of the existing dispatching misoperation prevention system were analyzed. A method about the integration between real-time data from dispatching SCADA system and data from substation misoperation prevention system was proposed, to solve the problem of incomplete high voltage equipment position in dispatching SCADA system; A method about the combination of the misoperation prevention check on instruction ticket and the online misoperation prevention locking on remote operation was proposed, to make up for the deficiency of the existing dispatching misoperation prevention system; A method about the hierarchical analysis on the grid topology was proposed, to improve the rate and efficiency of the online operation topology analysis. A novel dispatching misoperation prevention system was designed. The system has been put into application. The research result can be used as a reference for construction of integrated dispatching and controlling.
\end{abstract}

\section{Introduction}

The misoperation accidents are always one of the major risks in power grid operation. Major accidents due to operator misoperation have occurred frequently in power system. The research on the power grid dispatching operation misoperation prevention system began in the 80s of last century, some universities and research institutes have conducted a special study on this, developed some misoperation prevention system. In paper [1], the scheme focuses on the intelligent control of operation flow and instruction ticket; the scheme described in paper [2] does not concern the operation of the substation equipment such as "breaker, disconnector switch", only for the transition of large equipment state; the solution for dispatching operation misoperation prevention in paper [3-5] just stays at the software level. From the misoperation prevention system has been put into operation and the related papers, most of the existing researches focus on intelligent operation ticket system ${ }^{[6-8]}$ or dispatching operation flow management system. The successful development of these systems reduced the intensity of the work of dispatchers and ensured the correct rate of the dispatching operation tickets, at the same time, restricted the habitual violation of dispatcher through technical means, greatly reduced the dispatching misoperation. However, with the development of the integrated dispatching and controlling, the operation mode of traditional dispatch and substation have be changed, the original substation supervising and controlling, remote controlling, and other operations executed by dispatching center, existing dispatching misoperation prevention system does not meet the safety requirements.

Based on this, in order to adapt to China's power grid operation system of integrated dispatching and controlling, and minimize the dispatching misoperation, in this paper, research on dispatching misoperation prevention technology under the background of integrated dispatching and controlling was carried out, and on this basis, a new type of dispatching misoperation prevention system was designed. 


\section{General idea}

The system used open external interface, integrated real-time data of dispatching SCADA system and access data of substation misoperation prevention system, established panoramic data platform containing all substations high voltage equipment state signal, solved the problem that the state signal acquisition of the high voltage equipment of the dispatching SCADA system was not complete, achieved a comprehensive analysis of the misoperation prevention logic; Used misoperation prevention technology which combined misoperation prevention checking of instruction ticket with on-line misoperation prevention locking of remote operation, improved the limitations of existing dispatching misoperation prevention system, ensured the safety of the entire process of dispatching operations; Used hierarchical analysis technology according to bay layer, substation control layer, dispatching regional layer, improved the rate and efficiency of on-line security topology analysis, achieved real-time analysis of misoperation prevention.

According to the above ideas, a set of dispatching misoperation prevention system were designed, the functions included misoperation prevention, intelligent generating tickets, process management of dispatching order ticket, topology analysis of whole power network and Web publishing. The system adopted the topology analysis of the power grid wiring, used a variety of security misoperation prevention technology, locked and alarmed when the operation such as violating dispatching safety rules or affecting power grid safety appeared.

Used of open modular software system architecture. Each module was designed independently, without affecting each other, ensured the stability and reliability of the system itself, which laid the foundation for the misoperation prevention analysis of dispatching operation. System structure diagram is shown in Figure 1. Developed a powerful communication module to support serial, network communication, etc, for getting panoramic data which used by misoperation prevention analysis of dispatching operation. Real-time status of equipment was displayed through integrated graphics and database module. The intelligent operation ticket module supports manual, graphics, typical ticket and other generating mode, and has a perfect command ticket process management functions. Misoperation prevention checking and network topology analysis module were designed to complete misoperation prevention checking for instruction ticket and on-line misoperation prevention locking for remote operation.

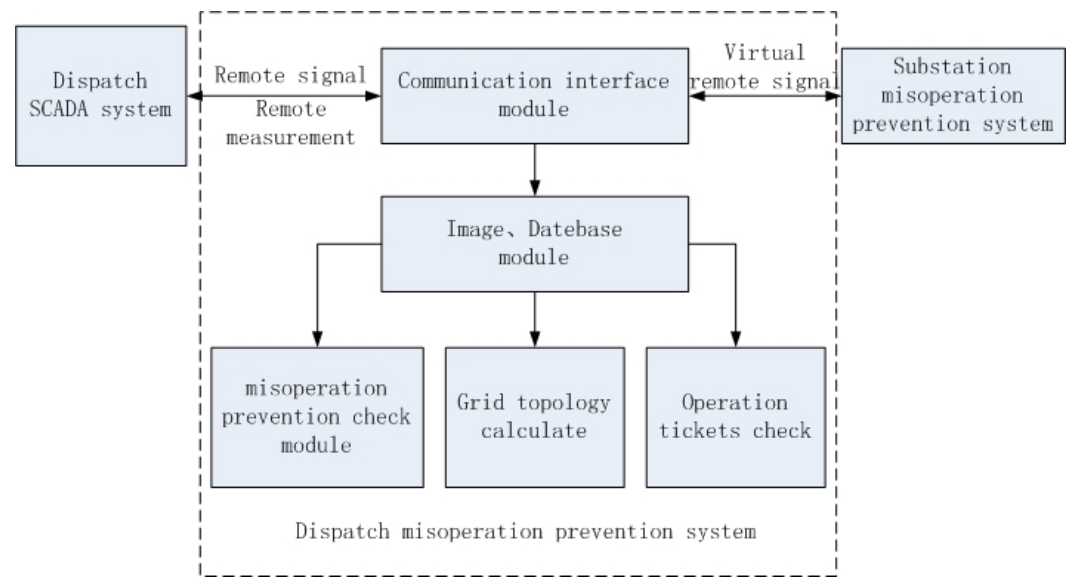

Fig.1 Structure diagram for dispatching misoperation prevention system

\section{Key Technologies}

\section{Panoramic data platform}

Most dispatching SCADA system did not collect the information such as net door, earthing wire, grounding switch, press plate state. The dispatcher lacked the safety assistance criterion for remote operation. The possibility of misoperation increased under the background of integrated dispatching and controlling. The dispatching misoperation prevention technology, based on real-time data of 
dispatching SCADA system and access data of substation misoperation prevention system, could solve this problem.

The system connected with dispatching SCADA system, used ethernet communication, TCP / IP protocol, IEC104 protocol, got real-time remote signal and measurement of high voltage equipment. In the technical implementation process, the reliability and security of data transmission in the dispatching data network were fully considered. The misoperation prevention host was isolated with dispatching SCADA system through the firewall, to ensure that the original data communication of dispatching was not affected. The system connected with substation misoperation prevention system, used of ethernet communications, TCP / IP protocol, fiber channel, used the mode including a server and a client. The misoperation prevention host of dispatching acted as a server, each operation and maintenance station and single substation acted as a client. The client would send the status information of the grounding switch, earthing wire, net door and pressure plate of substation to the server in real-time. Taking into account the various regions may exist in a variety of modes of operation (single substation, operation and maintenance station, etc.), all misoperation prevention data of substation could be sent to the dispatching and controlling center, to ensure the integrity of the data.

In order to ensure the collected substation data was correct and reliable, a temporary earthing wire management device ${ }^{[9]}$ was designed. The position signal of traditional earthing wire was sent by the style of virtual remote signal, this way could not accurately reflect the position of the earthing wire. Major misoperation accidents such as closing with earthing wire would occur very likely under the background of integrated dispatching and controlling. Therefore, it was important to collect the real-time location of the temporary earthing wire of the substation.

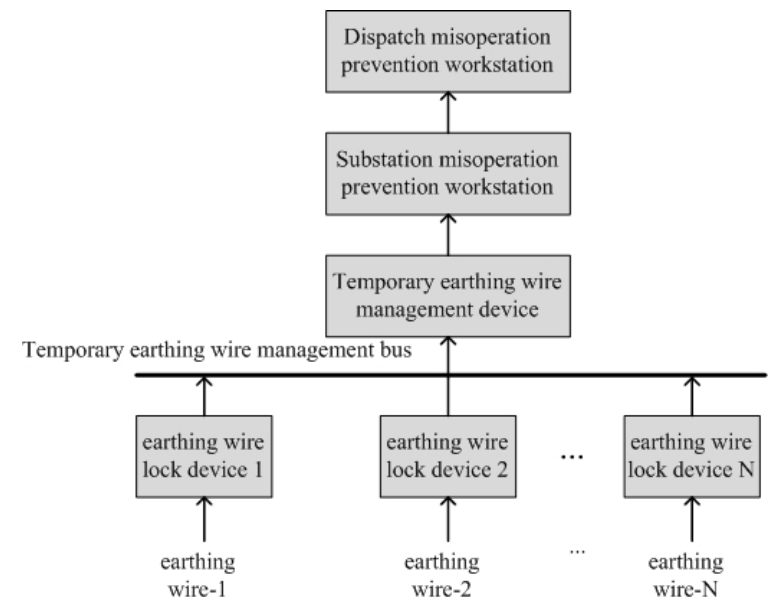

Fig.2 Structure diagram for collecting the real-time position of earthing wire

The structure diagram for the dispatching misoperation prevention system collecting the real-time position of earthing wire is shown in Figure 2. The position of earthing wire collected by the temporary earthing wire manager could be sent to dispatching misoperation prevention system by substation misoperation prevention system in real-time. The temporary earthing wire manager used the communication way of industrial field, to complete the information exchange between each temporary earthing wire lock which installed discretely. The communication way of industrial field bus, solved the problem of long-distance communication required in field installation of substations, achieved the requirement by one temporary earthing wire manager to control several temporary earthing wire locks. The interface between temporary earthing wire manager and substation misoperation prevention system supported serial, network and wireless and other mode.

\section{New technology of misoperation prevention}

In order to make up for deficiencies in the existing dispatching misoperation prevention system on the remote misoperation prevention, a scheme of misoperation prevention which combined misoperation prevention checking of instruction ticket with on-line misoperation prevention locking of remote operation was proposed. Used misoperation prevention checking technology for the dispatching 
instruction ticket, used misoperation prevention locking technology for remote control of dispatching operation.

Most of the existing dispatching misoperation prevention system used this model which established on the traditional dispatching and operation management. The traditional operation mode of dispatching is as follow : the chief dispatcher audited the dispatching instruction ticket after the deputy dispatcher have wrote the dispatching instruction ticket, the dispatching instruction ticket was sent to substation if the result of auditing was ok, operators in substation wrote operation ticket. In general, the dispatcher would sent dispatching instruction ticket to substation one day or more ahead of time to operate, however the power grid state was changing in real-time. If the operation mode of grid has changed or the equipment has appeared fault temporarily in the interval between the dispatching instruction ticket was sent to the substation and actual operation, the operator continues to operate in accordance with the dispatching instruction ticket which was defective, the operation may cause power grid accidents. When the technology of dispatching misoperation prevention checking of instruction ticket was applied, the life cycle of dispatching instruction ticket from writing to executing was checked and supervised, locked and alarmed when the operation such as violating dispatching safety rules or management process of dispatching instruction ticket. The technology can adapt to the traditional operation mode of dispatching, can effectively reduce the dispatching accident caused by fault of dispatching instruction ticket.

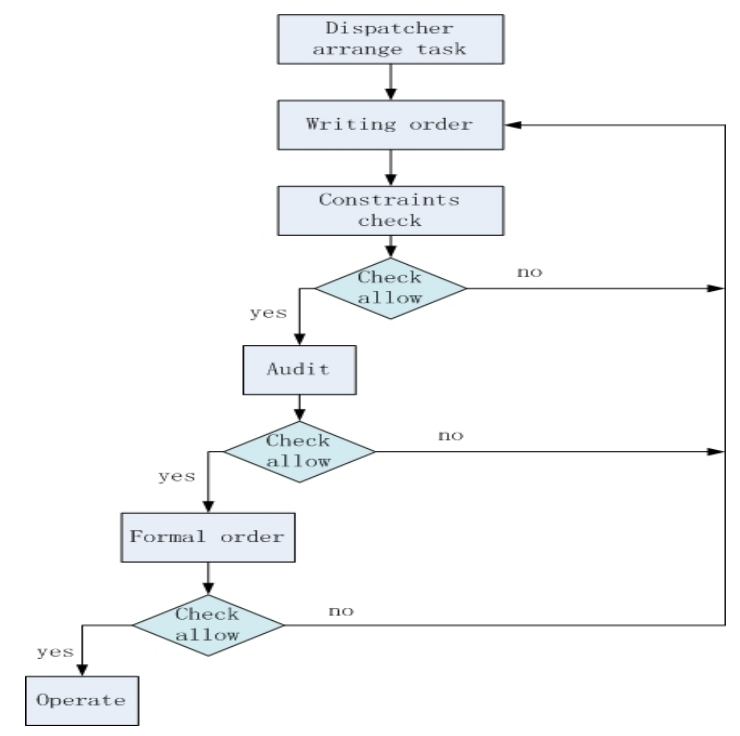

Fig.3 Check process of dispatching operation instruction

The process of misoperation prevention checking for dispatching operation instruction ticket is shown in Figure3. The dispatching operation instruction ticket was wrote after the dispatcher received a dispatching task, simulation operation and constraint check was executed in the process of writing ticket. The dispatching operation instruction ticket would be written again if constraint check did not pass, otherwise, the flow goes to the next step. In accordance with the step of writing ticket step, simulation operation and constraint check was executed before going into the process of audit, pre-order and formal order, the flow goes to the next step if constraint check pass, otherwise, the dispatching operation instruction ticket would be written again. The method solved the real-time problem of dispatching instruction ticket effectively, to prevent the defect of dispatching instruction ticket caused by operation mode change in the interval between writing and executing dispatching instruction ticket .

The technology of on-line misoperation prevention locking was applied when a remote control operation was executed in dispatching center. The technology would check the remote control request at the software layer of the dispatching side, would control the locking circuit of high voltage electrical equipment in substation directly. In this way, even if a wrong remote control instruction was sent by 
dispatching SCADA system, the high voltage electrical equipment also would not be action, as the lock unit on the locking circuit of equipment was still in the forced lock state.

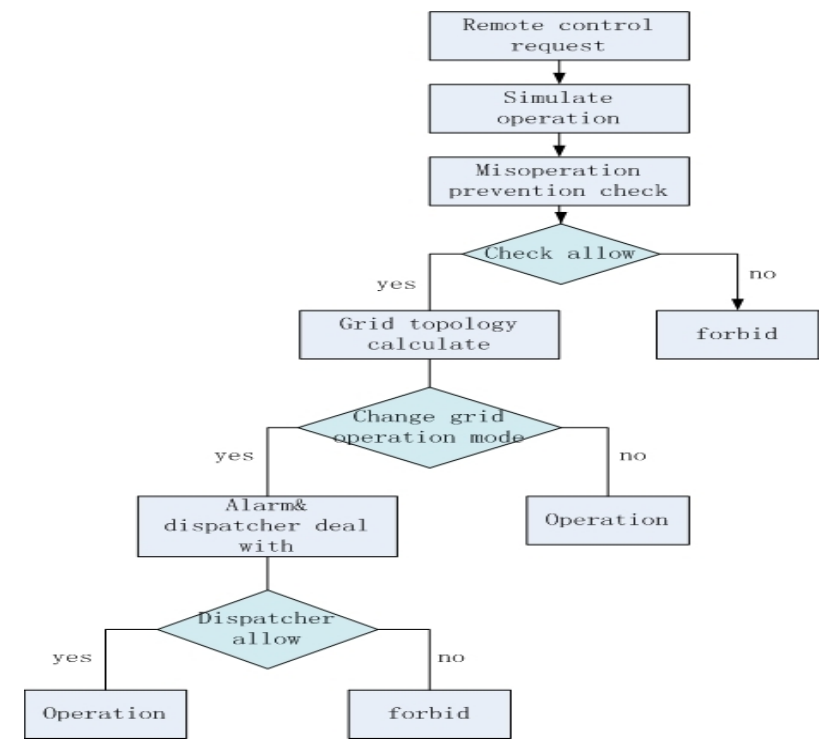

Fig.4 Misoperation prevention process of dispatching remote operation

The process of on-line misoperation prevention for dispatching operation is shown in Figure4. The dispatching misoperation prevention system executed simulation operation, and checked the remote control with misoperation prevention rules, after the remote control request of dispatching SCADA system has been received. If the remote control operation did not meet the misoperation prevention rules, the dispatching misoperation prevention system replied to prohibit remote control, maintained the lock state of locking unit simultaneously, otherwise, the dispatching misoperation prevention system executed grid topology calculate with the real-time remote signal and measurement, made judgments for the next step with the result of topology calculate. If the operation results changed the grid operation mode, such as loop closing, loop opening, or important users out of power, the system would alarm and display the result of calculating and the consequence of operation. The dispatcher was prompted to determine whether to proceed, if the dispatcher confirmed to continue the operation, the dispatching misoperation prevention system sent the permission reply to the dispatch SCADA system, at the same time, sent a unlock command to the locking unit; If the operation result did not change the grid operation mode, the dispatching misoperation prevention system checked by, allowed the remote operation, and unlock the lock unit.

\section{Hierarchical analysis of grid topology}

Rate and efficiency is the key to topology analysis ${ }^{[10-11]}$ of online operation safety. Based on the massive data and real-time refresh characteristics of dispatching center, the system applied the whole network topology analysis, if the operating mode of the field device changed during the analysis, the system need to recalculate the topology, which was computationally inefficient. The technology of hierarchical analysis of grid topology could solve the problem, the layer include bay, substation and dispatching region. 


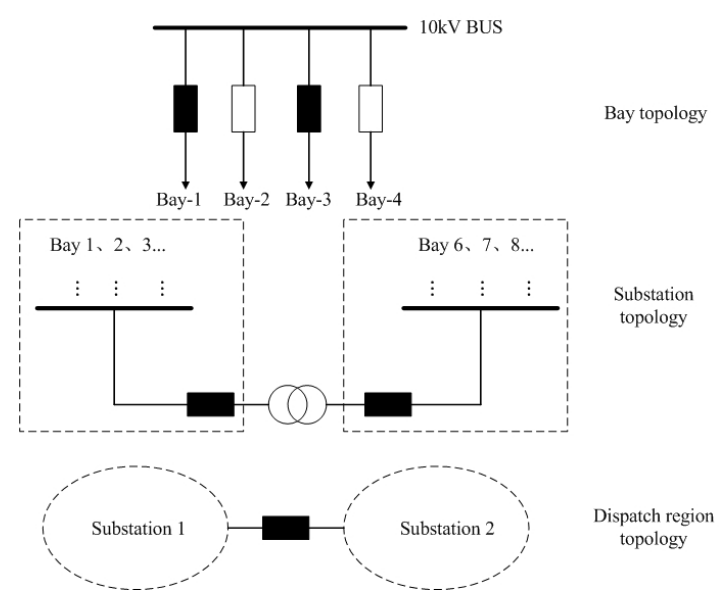

Fig.5 Hierarchical analysis for grid topology

The layer of hierarchical analysis of grid topology is shown in Figure 5. When the state of the field device changed, only the smallest classification area of topology was selected for calculation. For example, the operation of breaker in a $10 \mathrm{kV}$ circuit bay, the operation of the device would only have an impact on the state of the bay, therefore, only the bay topology was choose for the analysis calculation; In the case of the operation of breaker belong to transformer, the operation may be subject to running state of several bays in substation, the substation topology was choose for the analysis calculation; For equipment between two substation, such as contact switch, operation of the equipment would affect the relevant substation or a dispatching region, then the relevant dispatching region was choose for the analysis calculation. The technology adapt to massive data and real-time refresh characteristics of dispatching center, which can greatly improve the rate and efficiency of topology analysis while ensuring the accuracy of the analysis results.

\section{Conclusions}

Established panoramic data platform containing all substations high voltage equipment state signal; improved the limitations of existing dispatching misoperation prevention system, ensured the safety of the entire process of dispatching operations; improved the rate and efficiency of on-line security topology analysis, achieved real-time analysis of misoperation prevention.

The dispatching misoperation prevention system described in this paper has made remarkable achievement. The results can be used as a reference for the construction of integrated dispatching and controlling.

\section{References}

[1] Guo-qiang MU, Bin WEI, etal. Automation of Electric Power Systems, 2007, 31( 23 ):42-45. (In Chinese)

[2] Xing-ke ZHU, Biao BAI. Electric Power Automation Equipment, 2007, 27(5):96-100. (In Chinese)

[3] Prevost,Aubin Francois,Gauthier Jacques.Preventinghuman errors in power grid management systemsthrough user-interface redesign[C].2007 IEEE International Conference on Systems,Man,and Cybernetics,SMC2007,October7,2007-October 10,2007.

[4] Ramos, G.Sanchez J.L, Torres A. Power systems security evaluation using Petri nets[C].IEEE Transactions on Power Delivery, 2010, 25(1):316-322

[5] Burt G.M, McDonald J, R.Realtime decision support system for the operation of a $132 \mathrm{KV}$ power network[C].Proceedings of the 2nd InternationalConference on Advances in Power System Control, Operation \& Management, December 7, 1993-December 10, 1993 Sponsor: IEE. 
[6] Chuang-xin GUO, Chuan-bai ZHU, etal. Power System Technology, 2006, 30(4):50-54. (In Chinese)

[7] Ling QIAN, Feng GUO. Automation of Electric Power Systems, 2008, 32(23):100-103. (In Chinese)

[8] Shu-fang Chen, Kai Wang.Automation of Electric Power Systems, 2008, 32(15): 49-53. (In Chinese)

[9] Ren-qi Liu,Xiao-jun Lv,etal .Automation of Electric Power Systems,2010,22:109-112. (In Chinese)

[10]Nian Mei, Dong-yuan Shi, etal. Power System Technology, 2008, 32(13):35-39. (In Chinese)

[11]Bu-xiang Zhou, Xin-yu Liu .Automation of Electric Power Systems, 2003, 27(16):67-70. (In Chinese) 